This is an Author's Accepted Manuscript of an article published as: ROSETA-PALMA, C. and XEPAPADEAS, A. (2004) Robust Control in Water Management. Journal of Risk and Uncertainty, 29(1), 21-34 available online at:

http://dx.doi.org/10.1023/B:RISK.0000031443.39763.f0 


\title{
Robust Control in Water Management
}

\author{
Catarina Roseta-Palma* \\ Anastasios Xepapadeas ${ }^{\dagger}$
}

*Dinâmia and Dep. Economia - ISCTE, Av. Forças Armadas, 1649-026 Lisboa, Portugal, catarina.roseta@iscte.pt (corresponding author)

${ }^{\dagger}$ Dep. Economics, University of Crete

${ }^{\ddagger}$ We thank Maria Antonieta Cunha-e-Sá, Richard Woodward, Ronan Congar, Manuel Sequeira, Hans-Peter Weikard, and participants in the Sevilla Workshop on "Dynamic Economics and the Environment", as well as two anonymous referees, for many helpful comments and discussions. 


\begin{abstract}
Since surface water flows are often stochastic, there is a role for water reservoirs in protecting users against uncertainty. We assume uncertainty regarding the probability distribution for the stochastic variable. Thus the decision allows for a range of approximate models that could be true, and the problem can be solved using robust optimal control. This paper analyses the implications of a robust framework on resource management decisions, using the case of water as an illustration. Robust choices are compared with those of a benchmark stochastic model and the emergence of precautionary behaviour is discussed.

Keywords: Scientific Uncertainty, Maxmin Approach, Robust Control, Water Use, Rainfall Variability, Precaution

JEL classification: Q25, D81
\end{abstract}




\section{Introduction}

In most water systems there are multiple sources of water, with different availabilities and quality levels. A typical system will combine whatever surface water supplies are available (rainfall, stream flows, surface water reservoirs) between them and also with groundwater resources. Economic models of conjunctive use consider at least two sources of water, one of which is a flow and one a stock. For instance, the literature that analyses management of groundwater stocks normally includes conjunctive use (see the review on the topic by Provencher (1995)). Taking into account that surface water flows are often stochastic, there is a role to be played by groundwater or surface reservoirs in protecting users against uncertainty. Tsur (1990) studies the buffer role of groundwater in a static setting and shows that it is positive under standard concavity assumptions of the benefit function, and Tsur and Graham-Tomasi (1991) provide a similar analysis for a dynamic setting. Knapp and Olson (1995) also consider surface water variability, as well as Provencher and Burt (1994), which identify the risk externality associated with common property situations. RosetaPalma (2000) extends the analysis by incorporating water quality as a relevant parameter. There is a paper on irreversibility (Tsur and Zemel (1997)) where the size of stock below which groundwater use becomes unfeasible is unknown, but conjunctive use is not considered. Two other papers that consider uncertainty but not conjunctive use are Fisher and Rubio (1997) and Rubio and Castro (1996).

In all the literature referred so far, the word "uncertainty" is taken to describe the realization of an event for which the true probability distribution is known. Thus the expected utility framework can be used. However, this type of problem was traditionally considered one of risk. Pure uncertainty, where the state space of outcomes is known but the decision maker is unable to assign probabilities, has largely been ignored in recent economic literature. Nonetheless, as stressed by Woodward and Bishop (1997), in many cases pure uncertainty might be closer to the truth, for instance when a panel of experts is consulted, 
since a group of people with divergent beliefs will normally not be able to reach a consensus on probability distributions. Their paper analyses circumstances under which rational choices are based on the most extreme possible outcomes, rather than on midpoint values. It also discusses the intermediate case, where some information on the set of probability distributions is known. Gilboa and Schmeidler (1989) show that introducing an axiom of uncertainty aversion as a property of the preference relation is equivalent to solving a maxmin model under a set of possible probability measures, where the decision maker maximizes over the choice variables for the worst possible case. The construction of the appropriate set of measures, however, is not discussed.

Chevé and Congar (2000) consider situations of model uncertainty as well as risk (which they call imprecision and randomness, respectively). There is a cumulative pollution threshold beyond which a catastrophic event occurs, but knowledge of that threshold is modelled as a closed random interval. There is a class of random variables that are considered to be compatible with the available information, so that the chosen pollution path will depend on the decision maker's "optimism index".

Along similar lines, a dynamic approach for problems of choice under uncertainty is presented in Hansen and Sargent (2003) (discrete time setting), and Hansen et al. (2002) (continuous time setting). Again, the idea is that the decision maker is unsure about his model, in the sense that there is a group of approximate models that he also considers as possibly true. These are obtained by disturbing a benchmark model, and the admissible disturbances will reflect the set of possible probability measures that the decision maker is willing to consider. The resulting problem is one of robust dynamic control, where the objective is to choose a rule that will work under a range of different model specifications. This methodology provides another tractable way to incorporate uncertainty aversion.

The types of solutions obtained by models such as these fit quite well with the precautionary principle, which has emerged in interna- 
tional law as a conceptual guideline for environmental policy. ${ }^{1}$ In fact, Woodward and Bishop (1997) mention it explicitly, whereas Hansen and Sargent (2001a) state that "a preference for robustness induces context-specific precaution". More to the point, Chevé and Congar (forthcoming) stress the importance of scientific uncertainty in the interpretation and application of the precautionary principle. Considering the levels of uncertainty usually associated with climatic variables, it seems natural to exploit the instruments provided by robust control in the analysis of water storage and use decisions.

The purpose of our work is to show how a robust control framework of model misspecification doubts can be applied to resource management decisions. Uncertainty about the occurrence of precipitation is introduced, and the implications for quantitative water use are presented, in both a static and a dynamic setting. Robust choices are compared with those of a benchmark stochastic model, using a linear quadratic set up as a relevant representation of a system that comprises a surface storage water reservoir. Finally, the emergence of precautionary behavior is discussed and circumstances where robustness induces water savings are illustrated.

\section{A one period model of water management}

Assume that there is a user of water, who maximizes his profit by choosing the amount of water he wants. Precipitation $(P)$ is exogenous, but there is an available source of surface water (for example, a river), from which he can take as much water as he wants at a cost, given by a cost function $C(s)$, increasing and convex. There is a water revenue function which depends on total water use and is increasing and concave. Maximum profit, $\Pi$, is

$$
\Pi=\max _{s} Y(w)-C(s)
$$

\footnotetext{
${ }^{1}$ See Gollier, Julien and Treich (2000) for a discussion of the precautionary principle in an economy with a stock pollutant where there is learning about damages. The authors consider risk but not uncertainty aversion.
} 
where $w=P+s$, i.e. the total water usage is the sum of precipitation and pumping, making this model particularly suitable for irrigated agriculture.

It will be assumed that when the decision on $s$ is taken, the value for precipitation is still uncertain. In a typical stochastic problem, the agent would take $P$ to be a random variable with

$$
P=\bar{P}+\varepsilon
$$

where $\varepsilon$ has mean zero and variance $\sigma_{\varepsilon}^{2}=1$. In this very simple setting, the user maximizes the expected value of $\Pi$, and the first order condition will be $\mathcal{E} Y_{w}=C_{s}{ }^{2}$ This is the usual condition that at the optimal choice the expected marginal benefit of an additional unit of surface water is equal to its marginal cost.

However, it is possible that the agent views (2) as an approximation, in the sense that he is unsure about the process that governs the behavior of precipitation. ${ }^{3}$ One way to represent the uncertainty is to assume that he believes the process may be

$$
P=\bar{P}+\varepsilon+h
$$

where $h$ is an unknown distortion to the mean of the shock, representing a possible specification error.

The only thing that is assumed about this distortion is that the magnitude of the square of the specification error is bounded: ${ }^{4}$

$$
h^{2} \leq \eta^{2}
$$

Restriction (4), together with equation (3), define a set of models which the agent considers as being possible outcomes. Along the

\footnotetext{
${ }^{2}$ In order to study interior solutions, it is assumed that the last received unit of precipitation is still revenue increasing. Thus, floods and similar situations are ruled out.

${ }^{3}$ The derivations in this section are similar to Hansen and Sargent (2003, chp.5).

${ }^{4}$ The size of the distortion must be bounded, as the agent has some information on the process. For the restriction to be meaningful, $\eta$ must be finte. For a better explanation of this bound and its relation to the degree of uncertainty, see section 2 .
} 
lines of Gilboa and Schmeidler, the maximization will be undertaken considering the whole set of approximating models. The agent wants his decision to be robust, so that it will work well over the larger set, because he has doubts about the accuracy of the simpler approximation offered by equation 2 . He wants to choose a value for $s$ that will give him a reasonable outcome even for the worst possible value of $h$. Robust control provides a straightforward way to find optimal decisions that is relatively simple to solve. The uncertain problem can be thought of as a zero-sum game between two players, where the agent is maximizing over $s$ and nature is minimizing over $h$. Then the problem can be written as

$$
\max _{s} \min _{h} \mathcal{E}(Y(s+\bar{P}+\varepsilon+h))-C(s)+\theta h^{2}
$$

where $\theta>0$ is a fixed penalty parameter, which can be interpreted as a Lagrangian multiplier on constraint (4). ${ }^{5}$ First order conditions for $s$ and $h$, assuming an interior solution, are:

$$
\begin{aligned}
& \mathcal{E} Y_{w}=C_{s} \\
& \mathcal{E} Y_{w}=-2 \theta h
\end{aligned}
$$

\section{Quadratic case}

In order to understand how a preference for robustness influences optimal choices, this section presents the case where the objective function is quadratic. Water costs are assumed to be linear rather than strictly convex, because linearity is a more reasonable assumption for many distribution systems where pumping costs do not vary much and consequently unit costs are constant. With a quadratic production function, a unit price and linear costs, $Y=a+b w-c w^{2}$ and $C=e s$

\footnotetext{
${ }^{5}$ The value for $\theta$ would be endogenous in the constrained Lagrangian, and it would be associated to the specific $\eta$ value used in the constraint. The way the problem is written here, $\theta$ is chosen directly and the constraint is adapted accordingly. Larger values of $\theta$ imply smaller sets of models.
} 
(where $a, b, c$ and $e$ are positive parameters) and the approximating problem yields

$$
s^{*}=\frac{b-2 c \bar{P}-e}{2 c}
$$

If the agent is unsure about the model in the way described above, so that equation (2) is replaced with equation (3), then the corresponding problem yields:

$$
\begin{aligned}
s^{* *} & =\frac{b-2 c \bar{P}-e+\frac{c e}{\theta}}{2 c} \\
h^{* *} & =-\frac{e}{2 \theta}
\end{aligned}
$$

A few points can be made about the properties of this solution:

$h^{* *}$ is negative, i.e. the worst case distortion is a smaller mean for rainfall, as expected

when $\theta \rightarrow \infty, h^{* *} \rightarrow 0$ and $s^{* *} \rightarrow s^{*}$, i.e. as the agent becomes more sure about his model the solution tends to $(8)^{6}$

$s^{* *}>s^{*}$, i.e. in a one period model, if the agent is unsure about his model then his response will be to pump more. Precaution in this case implies excessive pumping, in the sense that the chosen level of surface water is optimal for the worst case $h$ and larger than would be optimal for all other values of $h$. This result is compatible with the usual observation that farmers, for instance, prefer to overirrigate if water is generally available (even though it is costly) in climates with great rainfall variability.

\footnotetext{
${ }^{6}$ Notice that the model breaks down if uncertainty aversion is infinitely large $(\theta \rightarrow 0)$.
} 


\section{Dynamic water management under model misspecifica- tion}

We now turn to the dynamic problem of managing surface water when the water manager is concerned about the robustness of his decisions to misspecification of the model, and there is accumulation of surface water in a reservoir.

Let $S_{t}$ denote the stock of surface water and let $\left\{B_{t}: t \geq 0\right\}$ denote a standard Brownian motion on an underlying probability space $(, \mathcal{F}, G)$. The water manager seeks to determine the optimal use of surface water. The manager's model can be stated as: ${ }^{7}$

$$
\begin{aligned}
\max _{\{s(t)\}} \mathcal{E} \int_{0}^{\infty} e^{-\delta t}\left[U\left(s_{t}+P_{t}\right)\right] d t \\
\text { subject to } \\
d S_{t}=\left(\alpha P_{t}-s_{t}-q S_{t}\right) d t \\
d P_{t}=\sigma d B_{t}
\end{aligned}
$$

where $U\left(s_{t}+P_{t}\right)=Y\left(s_{t}+P_{t}\right)-C(s), \delta$ is the discount rate, $\alpha$ is percentage of precipitation that ends up as stream flow, $q$ denotes losses from the surface water reservoir and $\sigma$ reflects precipitation variability.

Following Hansen and Sargent (2003), Hansen et al. (2002), (9)(11) is regarded as a benchmark model. If it was assumed that the water manager was sure about the benchmark model then there would be no concerns about robustness to model misspecification. Otherwise, concerns for robustness to model misspecification can be reflected by a family of stochastic perturbations to the Brownian motion, so that the probabilities implied by (11) are distorted. The measure $G$ is replaced by another probability measure $Q$. The main idea is that stochastic processes under $Q$ will be difficult to distinguish from those under $G$ using a finite amount of data. The perturbed model is constructed by

\footnotetext{
${ }^{7}$ The following analysis presents only interior solutions..
} 
replacing $B_{t}$ in (11) with

$$
B_{t}=\hat{B}_{t}+\int_{0}^{t} h_{s} d s
$$

where $\left\{\hat{B}_{t}: t \geq 0\right\}$ is a Brownian motion and $\left\{h_{t}: t \geq 0\right\}$ is a measurable drift distortion. Thus, changes in the distribution of $B_{t}$ will be parametrized as drift distortions to a fixed Brownian motion $\left\{\hat{B}_{t}: t \geq 0\right\}$. The distortions will be zero under the measure $G$, in which case $B_{t}$ and $\hat{B}_{t}$ coincide. ${ }^{8}$

Therefore, the water manager's concerns about misspecification of the model describing the evolution of precipitation can be expressed in the distorted model

$$
d P_{t}=\sigma h_{t} d t+\sigma d \hat{B}_{t}
$$

As shown in Hansen et al. (2002) the discrepancy between the distributions $G$ and $Q$ is measured as the relative entropy

$$
R(Q)=\int_{0}^{\infty} e^{-\delta u} \mathcal{E}_{Q}\left(\frac{\left|h_{u}\right|^{2}}{2}\right) d u
$$

If $R(Q)$ is finite then

$$
Q\left\{\int_{0}^{t}\left|h_{u}\right|^{2} d u<\infty\right\}=1
$$

and $Q$ is locally absolutely continuous with respect to $G$. Local absolute continuity means that for the water manager it is difficult to distinguish between the probability distributions $G$ and $Q$ associated with precipitation, even though the two probability distributions could be distinguished with infinite data. By restricting the size of relative entropy (14), the decision maker establishes the set of distributions that will be considered.

\footnotetext{
${ }^{8}$ For more general distortions, including changes in covariance, see Hansen and Sargent (2003, chp.17).
} 
Under model misspecification, equation (11) is replaced by (13). Two robust control problems can be associated with the problem of maximizing (9) subject to (10) and (13), a constraint robust control problem, which explicitly defines a relative entropy constraint, and a multiplier robust control problem. The multiplier robust problem in this case is defined as

$$
\begin{aligned}
& J(\theta)=\operatorname{maxmin}_{h} \mathcal{E} \int_{0}^{\infty} e^{-\delta t} U\left(s_{t}+P_{t}\right) d t+\theta R(Q) \\
& \text { subject to (10) and (13) }
\end{aligned}
$$

whereas the more intuitive constraint robust problem is defined as

$$
\begin{array}{r}
J(\eta)=\underset{s}{\operatorname{maxmin}_{h}} \mathcal{E} \int_{0}^{\infty} e^{-\delta t} U\left(s_{t}+P_{t}\right) d t \\
\text { subject to }(10),(13) \text { and } R(Q) \leq \eta
\end{array}
$$

In both models the process $\left\{h_{t}: t \geq 0\right\}$ belongs to a set $H$ such that the implied $Q$ has finite entropy or $R(Q)<\infty$. In the constraint model $\eta$ is the maximum specification error that the water manager is willing to accept, while in the multiplier model the robustness parameter can be interpreted as the Lagrangian multiplier associated with constraint $R(Q) \leq \eta$. The robustness parameter takes non negative values, $\theta \geq 0$, and will be zero if the constraint is inactive or infinity if the constraint is violated. A value $\theta=+\infty$ signifies no preference for robustness, while lower values for $\theta$ indicate such a preference.

Combining (14) and (15) the multiplier robust control model can be written as

$$
\begin{aligned}
& \underset{s}{\operatorname{maxmin}_{h}} \mathcal{E} \int_{0}^{\infty} e^{-\delta t}\left[u\left(s_{t}+P_{t}\right)+\theta \frac{h^{2}}{2}\right] d t \\
& \text { subject to (10) and (13) }
\end{aligned}
$$

As in section 1., in problem (17) the water manager is the maximizing agent that chooses surface water $s_{t}$ to maximize utility, while nature is the minimizing agent that chooses the worst case distortion 
to precipitation. Using Fleming and Souganidis (1989) on the existence of a recursive solution to the multiplier problem, Hansen et al. (2002) show that problem (17) can be transformed into a stochastic infinite horizon two-player game where the Bellman-Isaacs conditions imply that the value function $W(S, P, \theta)$ satisfies

$$
\delta W(S, P ; \theta)=\max _{s} \min _{h}\left\{\begin{array}{c}
{\left[u\left(s_{t}+P_{t}\right)+\theta \frac{h^{2}}{2}\right]+W_{S}\left(\alpha P_{t}-s_{t}-q S_{t}\right)} \\
+W_{P} \sigma h+\frac{1}{2} \sigma^{2} W_{P P}
\end{array}\right\}
$$

A solution for game (18) for any given value of the robustness parameter $\theta$ will determine the optimal "robust" surface water management policy. Moreover, this policy will coincide with the solution for the constraint problem (16) and it will not depend on the chosen timing protocol. Thus, the solution is time consistent, in the sense that the decision maker will stick to the original optimal plan whatever the actual state of the world. ${ }^{9}$

\section{Quadratic case}

Adopting a quadratic benefit function for net profits from water use, $U\left(s_{t}+P_{t}\right)$ is specified as

$$
U\left(s_{t}+P_{t}\right)=a+b\left(s_{t}+P_{t}\right)-c\left(s_{t}+P_{t}\right)^{2}-e s_{t}
$$

The first order conditions that determine the optimal feedback rules for $s$ and $h$ are:

$$
\begin{aligned}
s_{t}^{*} & =\frac{b-2 c P_{t}-e-W_{S}\left(S_{t}, P_{t} ; \theta\right)}{2 c} \\
h_{t}^{*} & =-\frac{\sigma W_{P}\left(S_{t}, P_{t} ; \theta\right)}{\theta}
\end{aligned}
$$

From (21) it is clear that if $\theta \rightarrow \infty$ then $h_{t}^{*} \rightarrow 0$ indicating that the manager acts as if he knows the model with certainty and there are no robustness concerns.

\footnotetext{
${ }^{9}$ For more on time consistency, see Hansen and Sargent (2001b).
} 
Substituting (20) and (21) into (18) we obtain the partial differential equation for the value function. Because of the linear quadratic structure of the problem we restrict our attention to the class of quadratic value functions, or

$$
W(S, P)=\gamma_{0}+\gamma_{1} S+\gamma_{2} S^{2}+\gamma_{3} P+\gamma_{4} P^{2}+\gamma_{5} S P
$$

Substituting (22), (20) and (21) into (18) we obtain the parameters of the value function, which can be solved for to yield a stable solution (see derivations in the Appendix): ${ }^{10}$

$$
\begin{aligned}
\gamma_{1} & =\frac{(2 q+\delta)\left(\theta \delta q(e-b)+2(1+\alpha) e \sigma^{2} c\right)}{\left(K-\theta q^{2}\right) \delta} \\
\gamma_{2} & =-(2 q+\delta) c q^{2} \frac{\theta}{K-\theta q^{2}} \\
\gamma_{3} & =\frac{\theta q^{3} e+(e-b) \theta \delta q(1+\alpha)(2 q+\delta)+B e(q+\delta)}{-q\left(K-\theta q^{2}\right) \delta} \\
\gamma_{4} & =-(2 q+\delta)(1+\alpha)^{2} c \frac{\theta}{K-\theta q^{2}} \\
\gamma_{5} & =2 q c(2 q+\delta)(1+\alpha) \frac{\theta}{K-\theta q^{2}}
\end{aligned}
$$

where $K=2 c \sigma^{2}(\alpha+1)^{2}>0$. For stability, $\gamma_{2}<0$, which in turn implies $\gamma_{4}<0$ and $\gamma_{5}>0$. The policy function can then be analyzed:

$$
s^{*}=\frac{1}{2 c}\left(b-\gamma_{1}(\theta)-e-\left(\gamma_{5}(\theta)+2 c\right) P-2 \gamma_{2}(\theta) S\right)
$$

Thus, in the robust problem, optimal water withdrawals depend positively on existing stock and negatively on precipitation, as expected. Moreover, increasing robustness (decreasing $\theta$ ) will make $s$ depend less on rainfall and more on stock (as $\frac{\partial \gamma_{5}}{\partial \theta}>0$ and $\frac{\partial \gamma_{2}}{\partial \theta}<0$ ). However, the full impact of increasing robustness on surface water applications will depend on the behaviour of the constant term, since

\footnotetext{
${ }^{10}$ The constant term is not presented as it has no impact on policy.
} 
$\frac{\partial \gamma_{1}}{\partial \theta} \gtrless 0$ depending on the sign of $(e-b)$. In a dynamic context where the accumulation of water is taken into consideration, a search for robustness can induce water savings. The water manager might decide to use less water as a precaution, since in a dynamic setting the worst case scenario would be to excessively deplete the stored water reserves. It should be noted, however, that the type of precautionary motive that was present in the static case, leading to an increase in water use, is still present here. Numerical simulations indicate that at higher levels of precipitation robustness results in more water application, whereas for lower levels of precipitation, the scarcity effect appears and robust decisions imply water savings.

The case that was just presented is particular in that stock only enters the problem as a restriction, not affecting the objective function directly. In a non-robust framework of optimization, this would imply that interior solutions are not affected by stock at all, and decisions for surface water applications would only depend on $P$ (so that all $\gamma$ coefficients are zero except the constant and $\gamma_{3}$, see Appendix).

A more general model, which would be adequate for groundwater reservoirs as well, would specifically include stock effects into water costs. The simplest possible type of stock effect, which is commonly used in models of reservoir management, is for unit pumping costs to decrease linearly with stock, so that $C(s, S)=C(S) s$ with $C(S)=C_{0}-C_{1} S$. The first order conditions that determine the optimal feedback rules for $s$ and $h$ are:

$$
\begin{aligned}
s_{t}^{*} & =\frac{b-2 c P_{t}-C(S)-W_{S}\left(S_{t}, P_{t} ; \theta\right)}{2 c} \\
h_{t}^{*} & =-\frac{\sigma W_{P}\left(S_{t}, P_{t} ; \theta\right)}{\theta}
\end{aligned}
$$

As unit pumping cost is linear in stock, the problem remains linear quadratic, so we can again use value function (22). However, the implied system of value function coefficients no longer has an analytical solution. To provide insight into the effects of robustness, a numerical 
solution is presented, using the following set of parameter values: ${ }^{11}$

$$
\begin{aligned}
& \alpha=0.4, b=6.316347, c=0.000348993, C_{0}=1, C_{1}=0.01 \\
& \sigma=90, \delta=0.05, a=16342.668, q=0.1
\end{aligned}
$$

Considering these values, some implied feedback rules for the optimal allocation of surface water are: ${ }^{12}$

$$
\begin{aligned}
& s_{t}^{*}\left(S_{t}, P_{t} \mid \theta=100\right)=7115.9-2.2698 P_{t}+1.8011 S_{t} \\
& s_{t}^{*}\left(S_{t}, P_{t} \mid \theta=500\right)=7321.8-2.4712 P_{t}+1.7796 S_{t} \\
& s_{t}^{*}\left(S_{t}, P_{t} \mid \theta \rightarrow \infty\right)=7616.7-2.6915 P_{t}+1.7717 S_{t}
\end{aligned}
$$

Again, surface water decreases with precipitation and increases with the amount of stored water. Furthermore, as $P_{t}$ increases, total water use $\left(s_{t}+P_{t}\right)$ decreases, more so for lower levels of uncertainty, as the $P$ coefficient is, in absolute value, larger than one and smaller for lower values of $\theta$. The optimal policy for two different stock levels is shown in figures 1 and 2 .

[Figure 1]

[Figure 2]

These figures show exactly the same result that was discussed earlier in the context of the model without stock effects, namely that robustness means saving water for low values of precipitation but using relatively more for high levels of precipitation.

\section{Conclusion}

In this paper we use robust control theory to explore the implications of uncertainty aversion for natural resource management, con-

\footnotetext{
${ }^{11}$ The production function parameters are for tomato production in Portugal in $\mathrm{kg} / \mathrm{ha}$ (Oliveira et al. (1996) ) Total water application in the experimental studies varies between 4000 and $12000 \mathrm{~m}^{3} / \mathrm{ha}$.All water units in the simulation are in $m^{3} / h a$, so that $S$ should be interpreted as the number of $m^{3}$ available to each $h a$ of irrigated land. Hydrological parameters are averages for southern Portugal, monetary values are in euro $/ \mathrm{m}^{3}$, and price is again assumed to be 1 euro $/ \mathrm{kg}$ to simplify. The simulations were run for one $h a$ using Mathematica.

${ }^{12}$ Simulations were run for additional values of $\theta$, with similar results.
} 
sidering specifically the case of water. The assumption that natural resources can follow stochastic processes which are not perfectly known to decision makers seems a better fit to what is observed in reality than the usual model of risk where all stochastic processes are known and agents take them as given in expected utility maximization.

Previous authors have pointed to the emergence of precautionary behavior when robust models are used. In the one period illustration for water management, precaution implies excessive use of surface water, as there are no future penalties for the use of such a source. When a dynamic setting is considered, the water manager incorporates the possible depletion of water reserves under worst case rainfall shortcomings, and precautionary behavior may then imply lower surface water applications if precipitation is low. It should also be noted that the usual certainty equivalence property of linear quadratic stochastic models no longer holds in robust optimal control.

In this paper, results for water use were derived with and without stock effects on cost, and presented for varying levels of model uncertainty, as expressed by levels of the penalty parameter $\theta$, to explore and illustrate the implications of this particular type of methodology. However, in general $\theta$ can be chosen according to different criteria. For instance, a level of acceptable detection error probability for distinguishing between the approximating model and the worst case model can be selected. More specifically, for each $\theta$ the associated detection error probability can be calculated for a given sample using the likelihood ratios when the approximating model generates the data and when the worst case model is true (see Hansen and Sargent (2003, chp. 13)). Nonetheless, the purpose of this paper was to identify the possibilities of the robust control methodology and its links to uncertainty aversion and the precautionary principle. Further research should be dedicated to analyzing detection error probabilities using real data samples and considering alternative criteria for choosing $\theta$, such as system stability.

Robust control methods are especially useful for dynamic systems that are highly unstable and where catastrophic events, once set in mo- 
tion, are difficult to revert. We believe that stochastic models in many areas of economics, and in environmental and resource economics in particular, should make more use of this methodology. Water management, especially under climate change uncertainty, is only one of the fields where the application of such methods could yield new insights.

\section{A Calculating value function coefficients}

A1. Without stock effects

In the linear quadratic case, the value function is given by

$$
W(S, P)=\gamma_{0}+\gamma_{1} S+\gamma_{2} S^{2}+\gamma_{3} P+\gamma_{4} P^{2}+\gamma_{5} S P
$$

Value function coefficients come from the following system of equations

$$
\begin{aligned}
& \delta \gamma_{0}=a+\frac{b^{2}}{4 c}+\frac{\gamma_{1}^{2}}{4 c}+\frac{E^{2}}{4 c}-\frac{E b}{2 c}-\frac{b \gamma_{1}}{2 c}+\frac{E \gamma_{1}}{2 c}-\frac{1}{2} \frac{\gamma_{3}^{2} \sigma^{2}}{\theta}+\sigma^{2} \gamma_{4} \\
& \delta \gamma_{1}=\frac{\left(E-b+\gamma_{1}\right) \gamma_{2}}{c}-\frac{\sigma^{2}}{\theta} \gamma_{3} \gamma_{5}-\gamma_{1} q \\
& \delta \gamma_{2}=\frac{\gamma_{2}^{2}}{c}-\frac{1}{2} \frac{\sigma^{2}}{\theta}\left(\gamma_{5}\right)^{2}-2 \gamma_{2} q \\
& \delta \gamma_{3}=\frac{\gamma_{1} \gamma_{5}-b \gamma_{5}+E \gamma_{5}}{2 c}+E+(1+\alpha) \gamma_{1}-2 \frac{\sigma^{2}}{\theta} \gamma_{3} \gamma_{4} \\
& \delta \gamma_{4}=\frac{\gamma_{5}^{2}}{4 c}+(1+\alpha) \gamma_{5}-2 \frac{\sigma^{2}}{\theta} \gamma_{4}^{2} \\
& \delta \gamma_{5}=\frac{\gamma_{2} \gamma_{5}}{c}+(1+\alpha) 2 \gamma_{2}-2 \frac{\sigma^{2}}{\theta} \gamma_{4} \gamma_{5}-\gamma_{5} q
\end{aligned}
$$

which can be solved beginning with the equations for $\gamma_{2}, \gamma_{4}$ and $\gamma_{5}$. Although there is more than one solution, we want a real solution that satisfies stability condition

$$
\frac{d \dot{S}}{d S}=\frac{\gamma_{2}(\theta)}{c}-q<0 .
$$


It can be shown that for this condition to be satisfied, in both real solutions of (28), $\gamma_{2}$ would need to actually be negative. Moreover, we can rule out the solution which corresponds to the positive root of the $\gamma_{2}$ equation as it is more likely to be unstable and/or to yield solutions which are not admissible (negative $s$ and/or positive $h$ ) Given the chosen expression for $\gamma_{2}$ (which is presented in the complete solution (23), in the paper), it should also be noted that there are implications on the acceptable values of $\theta$, since $\gamma_{2}<0$ iff $2 c \sigma^{2}(\alpha+1)^{2}-\theta q^{2}>0$. For $\sigma$ very small, $\theta$ has to be either small or infinity (non-robust solution). The non-robust solution in this case has $\gamma_{1}=0, \gamma_{2}=0, \gamma_{5}=0, \gamma_{4}=0$, and $\gamma_{3}=\frac{e}{\delta}, \gamma_{0}=\frac{1}{4} \frac{4 a c+(e-b)^{2}}{\delta c}$, corresponding to a policy function. $s_{t}^{*}=$ $\frac{1}{2 c}(b-2 c P-e)$ which is exactly the same as in the static case.

\section{A2. With stock effects}

The system of value function coefficients for this case, assuming that $C(S)$ is linear, is given by (28) in the paper. For a given set of parameter values, the solution can be attained using numerical simulation. Although for each value of $\theta$ several different solutions can appear in Mathematica, depending on the initial guesses for the $\gamma$ coefficients, the solution we show in the paper is the one that correctly approaches the $\theta \rightarrow \infty$ solution, which corresponds to the negative root of the $\gamma_{2}$ equation of the following simpler system: 


$$
\begin{aligned}
& \delta \gamma_{1}=-\frac{1}{2}\left(\frac{\left(C_{1}-2 \gamma_{2}\right)\left(C_{0}-1+\gamma_{1}\right)}{c}\right)-\gamma_{1} q \\
& \delta \gamma_{2}=\frac{1}{4 c} C_{1}^{2}-\left(\frac{C_{1}}{2 c}+q\right) 2 \gamma_{2}+\frac{\gamma_{2}^{2}}{c} \\
& \delta \gamma_{3}=\frac{-b+C_{0}+\gamma_{1}}{2 c} \gamma_{5}+C_{0}+\gamma_{1}(1+\alpha) \\
& \delta \gamma_{4}=\frac{\gamma_{5}^{2}}{4 c}+\gamma_{5}(\alpha+1) \\
& \delta \gamma_{5}=-\left(\frac{C_{1}}{2 c}+q\right) \gamma_{5}-C_{1}+\frac{\gamma_{5} \gamma_{2}}{c}+2 \gamma_{2}(\alpha+1)
\end{aligned}
$$




\section{References}

Chevé, M. and Congar, R. (2000), 'Optimal pollution control under imprecise environmental risk and irreversibility', Risk Decision and Policy 5, 151-164.

Chevé, M. and Congar, R. (forthcoming), 'La gestion des risques environmentaux en présence d'incertitudes et de controverses scientifiques: Une interprétation du principe de précaution', Revue Économique .

Fisher, A. and Rubio, S. (1997), 'Adjusting to climate change: Implications of increased variability and asymmetric adjustment costs for investment in water reserves', Journal of Environmental Economics and Management 34, 207-227.

Fleming, W. H. and Souganidis, P. E. (1989), 'On the existence of value functions of two-player, zero sum stochastic differential games', Indiana University Mathematics Journal pp. 293-314.

Gilboa, I. and Schmeidler, D. (1989), 'Maximin expected utility with non-unique prior', Journal of Mathematical Economics 18, 141153.

Gollier, C., Julien, B. and Treich, N. (2000), 'Scientific progress and irreversibility : An economic interpretation of the "precautionary principle"', Journal of Public Economics 75, 229-253.

Hansen, L. and Sargent, T. (2001a), 'Acknowledging misspecification in macroeconomic theory', on the Web.

URL: ftp://zia.stanford.edu/pub/sargent/webdocs/research/costa6.pdf

Hansen, L. and Sargent, T. (2001b), 'Time inconsistency of robust control?', on the Web.

URL: ftp://zia.stanford.edu/pub/sargent/webdocs/research/uncertnote9.pdf 
Hansen, L. and Sargent, T. (2003), 'Robust control and model uncertainty in macroeconomics', on the Web.

URL: ftp://zia.stanford.edu/pub/sargent/webdocs/research/rgamesb.pdf

Hansen, L. et al. (2002), 'Robustness and uncertainty aversion', on the Web.

URL: http://home.uchicago.edu/lhansen/uncert12.pdf

Knapp, K. and Olson, L. (1995), 'The economics of conjunctive groundwater management with stochastic surface supplies', Journal of Environmental Economics and Management 28, 340-356.

Oliveira, A. et al. (1996), Análise econométrica de ensaios de rega na cultura do tomate, in N. S. de Carvalho et al., eds, 'Investigação Técnico-Económica Para a Promoção Da Competitividade Da Agricultura No Alentejo', Vol. E10, INIA-EAN, pp. 109-115.

Provencher, B. (1995), Issues in the conjunctive use of surface water and groundwater, in D. Bromley, ed., 'Handbook of Environmental Economics', Blackwell.

Provencher, B. and Burt, O. (1994), 'A private property rights regime for the commons: The case for groundwater', American Journal of Agricultural Economics 76, 875-888.

Roseta-Palma, C. (2000), Essays in Natural Resource Economics, PhD thesis, Faculdade de Economia da Universidade Nova de Lisboa.

Rubio, S. and Castro, J. (1996), 'Long-run groundwater reserves under uncertainty', Investigaciones Economicas XX(1), 71-88.

Tsur, Y. (1990), 'The stabilization role of groundwater when surface water supplies are uncertain: The implications for groundwater development', Water Resources Research 26(5), 811-818.

Tsur, Y. and Graham-Tomasi, T. (1991), 'The buffer value of groundwater with stochastic surface water supplies', Journal of Environmental Economics and Management 21, 201-224. 
Tsur, Y. and Zemel, A. (1997), On event uncertainty and renewable resource management, in D. Parker and Y. Tsur, eds, 'Decentralization and Coordination of Water Resource Management', Kluwer Academic Publishers.

Woodward, R. and Bishop, R. (1997), 'How to decide when experts disagree: Uncertainty-based choice rules in environmental policy', Land Economics 73, 492-507. 